\title{
Clinical Effect of Yiqi Naoluo Tong Decoction in the Treatment of Cerebral Infarction
}

\author{
Yujin $\mathrm{Hou}^{1, \mathrm{a}}$, Xiaosu $\mathrm{Jie}^{1}$, Xin Guo ${ }^{1}$, Yingying Zhang ${ }^{1}$, Hua Shi ${ }^{1}$, Yuanjun Lou ${ }^{1}$ \\ ${ }^{1}$ Henan Provincial Hospital of Traditional Chinese Medicine, Zhengzhou, Henan, 450002
}

\begin{abstract}
Objective: To apply Yiqi Naoluo Tong Decoction for clinical treatment of patients with cerebral infarction, and analyze the therapeutic effect of this method. Methods: 150 cases of cerebral infarction patients who were treated in our hospital were set as the research objects and divided into two groups. The conventional treatment methods were combined with Yiqi Naoluo Tong Decoction and single conventional treatment.

Results: After treatment, the improvement of the test group of patients was better than that of the control group, and compared with the control group, the comparison result showed that $\mathrm{P}<0.05$, the difference was significant and statistically meaningful.

Conclusion: The addition of Yiqi Naoluo Tong Decoction in the clinical treatment of patients with cerebral infarction can improve the patients' neurological deficits, quality of life, limb motor function and ability of daily living. This shows that this medicine has a higher clinical application value.
\end{abstract}

\section{Introduction}

Cerebral infarction is a relatively common disease in neurology. It has the characteristics of rapid onset and rapid development, so the mortality and disability rate are relatively high ${ }^{[1]}$. Most patients with this disease are middle-aged and elderly, but they have gradually become younger in recent years, which can cause more and more patients to seriously reduce their quality of life, and even threaten their life safety. Therefore, timely and effective treatment of cerebral infarction patients is very important $t^{[2]}$. According to the current situation, the most widely used clinical treatment methods are all western medicine treatments. However, if western medicine is used for a long time, it may cause damage to the patient's body mechanism and disturb the homeostasis. Therefore, it is necessary to try to apply Chinese medicine treatment in the clinical treatment of patients with cerebral infarction ${ }^{[3]}$. In this study, patients with cerebral infarction were set as the research object, and conventional treatment methods were combined with Yiqi Naoluo Tong Decoction and single conventional treatment. The report is as follows.

\section{Materials and methods}

\subsection{General information}

150 cases of cerebral infarction patients who were treated in our hospital were set as the research objects. Admission time: February 2019-February 2020; inclusion criteria: in line with the clinical diagnostic criteria of cerebral infarction in our hospital and able to actively cooperate with the study; exclusion criteria : patients with mental disorders, patients with kidney disease, and patients with cardiopulmonary insufficiency; grouping method: digital odd and even number mode; group: test group (male to

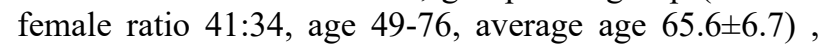
control group (male to female ratio 42:33, age 50-75 years, average age $64.5 \pm 6.3$ years). The general data of all aspects of 150 study subjects $P>0.05$, and this study in our hospital has been approved by the ethics committee.

\subsection{Treatment methods}

\subsubsection{Treatment methods for patients in the control group}

Give patients oxygen, keep the airway unobstructed, then maintain nutrition and dehydration, next lower intracranial pressure, finally control blood pressure and blood sugar, etc., take aspirin once every night, each time $0.1 \mathrm{~g}$. If the patient develops an infection, antibiotics can be used for treatment ${ }^{[4]}$.

\subsubsection{Treatment methods for patients in the test group}

On the basis of the treatment method of the control group, the test group was treated with Yiqi Naoluo Tong Decoction. The prescriptions were Astragalus 50g, Angelica 15g, Sichuan Achyranthes bidentata 15g, Peach kernel 9g, Safflower 9g, Leech 9g, Alisma 9g, mulberry 
branch $9 \mathrm{~g}$, chuanxiong $12 \mathrm{~g}$, red peony $12 \mathrm{~g}$, tuckahoe $12 \mathrm{~g}$, rice kernel $30 \mathrm{~g}$, whole scorpion $3 \mathrm{~g}$, stiff silkworm $6 \mathrm{~g}$, chonnan star $6 \mathrm{~g}$. Decocting them in water into $400 \mathrm{ml}$ juice, and taking 1 dose a day in the morning and evening with continuous medication for 8 weeks ${ }^{[5]}$.

\subsection{Observation indicators}

(1) Comparison of NIHSS neurological deficit score; (2) Comparison of QOL quality of life score and FMA limb motor function score; (3) Comparison of ADL daily living ability score.

\subsection{Statistical methods}

Using SPSS 20.0 to process and analyze the data of this study. The data on this study are all measurement data, which should be represented by $(\bar{x} \pm s)$ and verified by the $\mathrm{T}$ value. If the comparison between groups shows
$\mathrm{P}<0.05$, it shows that there is a significant difference in the comparison and it is statistically meaningful.

\section{Results}

\subsection{Comparison of the NIHSS neurological deficit score between the two groups}

Before treatment, the scores of the two groups of patients were $\mathrm{P}>0.05$. After treatment, the test group had consciousness score $(2.53 \pm 0.78)$, gazing score $(5.71 \pm 0.90)$, visual field score $(7.44 \pm 1.27)$, facial paralysis score(10.36 \pm 1.30$)$, upper limb movement score $(12.25 \pm 2.08) \quad, \quad$ lower limb movement score(13.17 \pm 2.36$)$, limb coordination score(15.48 \pm 2.25 ), compared with the control group patients' scores, the comparison result shows $\mathrm{P}<0.05$, the difference is significant and statistically meaningful. See Table 1

Table1. Comparison of NIHSS neurological deficit scores between the two groups $(\bar{x} \pm s)$

\begin{tabular}{ccccc}
\hline Group & Test group $(\mathbf{n = 7 5})$ & $\begin{array}{c}\text { Control group } \\
(\mathbf{n = 7 5})\end{array}$ & t & P \\
\hline consciousness & $2.53 \pm 0.78$ & $12.03 \pm 2.07$ & 11.452 & $<0.05$ \\
gazing & $5.71 \pm 0.90$ & $13.15 \pm 3.30$ & 10.067 & $<0.05$ \\
visual field & $7.44 \pm 1.27$ & $14.27 \pm 3.79$ & 12.334 & $<0.05$ \\
facial paralysis & $10.36 \pm 1.30$ & $15.13 \pm 4.22$ & 10.720 & $<0.05$ \\
upper limb movement & $12.25 \pm 2.08$ & $15.59 \pm 4.78$ & 11.542 & $<0.05$ \\
lower limb movement & $13.17 \pm 2.36$ & $16.34 \pm 5.64$ & 10.420 & $<0.05$ \\
limb coordination score & $15.48 \pm 2.25$ & $17.50 \pm 6.25$ & 11.427 & $<0.05$ \\
\hline
\end{tabular}

\subsection{Comparison of QOL quality of life score and FMA score between the two groups}

After treatment, the QOL quality of life score of patients in the test group was $(85.45 \pm 6.07)$, and the FMA limb motor function score was $(45.36 \pm 10.29)$. Compared with the scores of the control group, the comparison results showed that $\mathrm{P}<0.05$, the difference was significant and statistically meaningful. See Table 2 .

Table2. Comparison of QOL quality of life score and FMA limb motor function score between the two groups ( $\bar{x} \pm s$ )

\begin{tabular}{ccccc}
\hline \multirow{2}{*}{ Group } & \multicolumn{2}{c}{ Before treatment } & \multicolumn{2}{c}{ After treatment } \\
\cline { 2 - 5 } & $\begin{array}{c}\text { QOL } \\
\text { Score }\end{array}$ & $\begin{array}{c}\text { FMA } \\
\text { Score }\end{array}$ & $\begin{array}{c}\text { QOL } \\
\text { Score }\end{array}$ & FMA Score \\
\hline $\begin{array}{c}\text { Test group } \\
(\mathrm{n}=75)\end{array}$ & $64.58 \pm 5.20$ & $32.39 \pm 9.26$ & $85.45 \pm 6.07$ & $45.36 \pm 10.29$ \\
$\begin{array}{c}\text { Control group } \\
(\mathrm{n}=75)\end{array}$ & $63.68 \pm 5.16$ & $31.34 \pm 7.14$ & $69.89 \pm 4.25$ & $43.74 \pm 8.53$ \\
$\mathrm{t}$ & 3.465 & 2.773 & 11.592 & 13.708 \\
$\mathrm{P}$ & $>0.05$ & $>0.05$ & $<0.05$ & $<0.05$ \\
\hline
\end{tabular}

\subsection{Comparison of ADL scores between two groups}

Before 30 days of treatment, the ADL score of daily living ability of the test group was (55.48 \pm 6.89$)$, and after 3 months of treatment, it was $(73.15 \pm 7.43)$, and the total difference was $(53.64 \pm 4.67)$, compared to that of the control group. The comparison results show that $\mathrm{P}<0.05$, the difference is significant and statistically meaningful. See Table 3. 
Table3. Comparison of ADL scores between the two groups of patients ( $\bar{x} \pm s$ )

\begin{tabular}{ccccc}
\hline Group & Before treatment & $\begin{array}{c}\text { 30 days after } \\
\text { treatment }\end{array}$ & $\begin{array}{c}\text { 3 months after } \\
\text { treatment }\end{array}$ & Total difference \\
\hline $\begin{array}{c}\text { Test group } \\
(\mathrm{n}=75)\end{array}$ & $25.14 \pm 5.34$ & $55.48 \pm 6.89$ & $73.15 \pm 7.43$ & $53.64 \pm 4.67$ \\
$\begin{array}{c}\text { Control group } \\
(\mathrm{n}=75)\end{array}$ & $25.66 \pm 4.89$ & $50.56 \pm 5.25$ & $61.25 \pm 8.55$ & $41.75 \pm 5.65$ \\
$\mathrm{t}$ & 2.945 & 11.503 & 12.149 & 10.772 \\
$\mathrm{P}$ & $>0.05$ & $<0.05$ & $<0.05$ & $<0.05$ \\
\hline
\end{tabular}

\section{Discussion}

Traditionally, patients with cerebral infarction were treated with western medicine. In this study, the treatment of Chinese medicine was added to the treatment process, which is helpful to accelerate the treatment process of patients ${ }^{[6]}$. This study showed that before treatment, the scores of the two groups of patients were $\mathrm{P}>0.05$. After treatment, the test group showed the following score result: consciousness (2.53 \pm 0.78$)$, gazing $(5.71 \pm 0.90)$, visual field $(7.44 \pm 1.27)$, facial paralysis $(10.36 \pm 1.30)$, upper limb movement $(12.25 \pm 2.08)$, lower limb movement (13.17 \pm 2.36$)$, limb coordination $(15.48 \pm 2.25)$, QOL quality of life $(85.45 \pm 6.07)$, FMA limbs motor function (45.36 \pm 10.29$)$, ADL daily living ability (55.48 \pm 6.89$)$ after 30 days of treatment, and $(73.15 \pm 7.43)$ after 3 months of treatment. The total score difference was $(53.64 \pm 4.67)$ compared with the scores of patients in the control group, the comparison results showed that $\mathrm{P}<0.05$, the difference is significant and statistically meaningful. The results of this study are basically the same as those of scholar Xiaoli Zhao ${ }^{[8]}$.

The results of the study showed that the conditions of the test group were better than those of the control group, thus effectively showing the effect of Yiqi Naoluo Tong Decoction on patients with cerebral infarction. In Yiqi Naoluo Tong Decoction, Astragalus has the effect of replenishing qi and dredging pulse, which can reduce the viscosity of the patient's blood and reduce the chance of thrombosis ${ }^{[7]}$. Angelica can lower blood pressure and regulate blood lipids. Salvia can promote blood circulation and disperse blood stasis, it can also prevent platelet aggregation, and effectively regulate the patient's internal and external blood coagulation system.

In summary, the addition of Yiqi Naoluo Tong Decoction in the clinical treatment of patients with cerebral infarction can improve the patient's neurological deficits, quality of life, limb motor function, and daily life ability, which shows that this medicine has a high clinical application value.

\section{Acknowledgement}

At this point, the entire paper has ended. In the process of conducting this research and writing this paper, my work partner gave me a lot of encouragement, support and help. The research subjects and the patients' family members also communicated patiently and meticulously with me as much as possible, which not only benefits me to gain a indepth control of the information, but also give me enough motivation to work. In addition, many work leaders, seniors, and colleagues have reached out to help me, which has become an important foundation for my smooth work. Therefore, I would like to express my heartfelt thanks to all the people above.

\section{References}

1. Yanhui Liu, "Effect of Yiqi Huoxue Huatan Tongluo Decoction on TCM Syndrome Score and Cerebral Hemodynamics in Patients with Cerebral Infarction in the Recovery Period (Qi Deficiency and Blood Stasis Type)" [J]. Capital Food and Medicine, 2020, 27( 11):198.

2. Zhihua Liao, "Observation on the clinical efficacy of Yiqi Huoxue Tongluo Decoction in the treatment of Qi deficiency and blood stasis syndrome during the recovery period of cerebral infarction" [J]. Forum on Primary Medicine, 2020, 24(5): 706-708.

3. Jinxue Wang, "The clinical efficacy of Yiqi Huoxue Tongluo Decoction combined with Rosuvastatin in the treatment of acute cerebral infarction and its influence on related biochemical indicators" [J]. Chinese Folk Therapy, 2020, 28(3): 60-62.

4. Maolin Liu, Dayong Lin, Jian Bai, "The effect of Yiqi Huoxue Tongluo Decoction in the treatment of acute cerebral infarction and its influence on limb dysfunction" [J]. Chinese Journal of Traditional Chinese Medicine, 2019, 37(5): 1234-1236.

5. Qingyu Yang, Rui Zhao, "Effect of Yiqi Huoxue Tongluo Decoction on neurological deficit and serum glial fibrillary acidic protein, Ang- II and VEGF levels in patients with cerebral infarction" [J]. Information on Traditional Chinese Medicine, 2019, 36(1) ): 94-98.

6. Hongyan Kang, "Analysis of the clinical effect of Yiqi Huoxue Huatan Tongluo Decoction combined with injection of brain protein hydrolysate in the treatment of acute cerebral infarction" [J]. Clinical Medical Research and Practice, 2018, 3(7): 126-127.

7. Qian Yang, "Clinical study of Yiqi Huoxue Huatan Tongluo Decoction combined with ozagrel sodium in the treatment of cerebral infarction with Qi deficiency, blood stasis and phlegm obstruction"'[J]. Asia-Pacific Traditional Medicine, 2017, 13(21): 144-145. 
8. Xiaoli Zhao, "Effects of Buzhong Yiqi Decoction and Shenma Tongluo Capsules on Hemorheology and Blood Lipids in the Recovery Period of Middle-aged Cerebral Infarction"[J]. Exploration of Rational Drug Use in China, 2017, 14(7): 23- 25, 42. 